Rev. Téc. Ing. Univ. Zulia. Vol. 42, No. 2, 2019, 76-85

\title{
Prediction of citral superficial content on microparticles by NIR spectroscopy and partial least square regression
}

\author{
I. Yoplac ${ }^{1,2^{*}}$, H. Avila-George ${ }^{3}$, L. Vargas ${ }^{4}$, P. Robert ${ }^{5}$, W. Castro ${ }^{6}$ \\ ${ }^{1}$ Facultad de Ingeniería Zootecnista, Agronegocios y Biotecnología, Universidad Nacional Toribio Rodríguez de Mendoza de \\ Amazonas. Chachapoyas, Perú. \\ ${ }^{2}$ Escuela de Postgrado, Programa Doctoral en Ciencia de Alimentos, Universidad Nacional Agraria La Molina, Perú. \\ ${ }^{3}$ Centro Universitario de los Valles, Universidad de Guadalajara. Ameca, Jalisco 46600, México. \\ ${ }^{4}$ Facultad de Industrias Alimentarias, Universidad Nacional Agraria La Molina, Perú. \\ ${ }^{5}$ Dpto. Ciencia de los Alimentos y Tecnología Química, Facultad de Ciencias Químicas y Farmacéuticas, Universidad de Chile, \\ Casilla 133, Santiago, Chile. \\ ${ }^{6}$ Facultad de Ingeniería, Universidad privada del Norte, Cajamarca, 06001, Perú. \\ *Autor de Contacto: ives.yoplac@untrm.edu.pe \\ https://doi.org/10.22209/rt.v42n2a04 \\ Recepción: 31/01/2019 | Aceptación: 07/03/2019 | Publicación: 01/05/2019
}

\begin{abstract}
In this study NIR spectroscopy and Partial Least Square Regression (PLSR) were evaluated for prediction of citral superficial content (SC-superficial of citral) on microparticles. For this purpose, samples of microparticles with different SC were prepared; varying the citral:dextrin (Ct:Dx) ratio, in the solution (1:5 and 1:20), in the solution and inlet air temperature (TAI) in a spray dryer $\left(120\right.$ and $\left.200{ }^{\circ} \mathrm{C}\right)$, in the drying process, getting 12 treatments. The SC was measured by gas chromatography. Then, each treatment was divided into six-parts, four for modeling and two for validation, obtaining 72 parts and from each one its absorbance spectral profile (1100 and $2500 \mathrm{~nm}$ ), was extracted and pretreated. Subsequently, the PLSR method was applied to model the relationship between the spectral profiles and the SC values. Models were obtained with full spectra and optimized models with the relevant wavelengths. The results obtained with the optimized PLSR model showed R2 values higher than 0.89 and RMSEC less than 4.86x10-15. So, the NIR-PLSR method showed potential for the determination of citral on microcapsules.
\end{abstract}

Keywords: Absorbance; Encapsulation efficiency; Microencapsulation; Spectral profile; Reflectance.

\section{Predicción del contenido superficial de citral en micropartículas mediante espectroscopía NIR y regresión por mínimos cuadrados parciales}

\section{Resumen}

\begin{abstract}
En este estudio se evaluó la espectroscopía NIR y la regresión de mínimos cuadrados parciales (PLSR - partial least square regression) en la predicción del contenido superficial de citral (SC- superficial de citral) en micropartículas. Para este propósito, se prepararon muestras de micropartículas con diferentes niveles de SC variando la relación citral: dextrina (Ct:Dx) en la solución (1:5 y 1:20), y la temperatura del aire de ingreso (TAI) al secador por atomización (120 y $\left.200{ }^{\circ} \mathrm{C}\right)$ durante el secado, obteniendo 12 tratamientos. El contenido SC se midió por cromatografía de gases. Luego, cada tratamiento se dividió en seis partes, cuatro para modelado y dos para validación, obteniendo 72 partes de las cuales su perfil espectral de absorbancia (1100 y $2500 \mathrm{~nm}$ ), fue extraído y pretratado. Posteriormente, se aplicó el método PLSR para modelar la relación entre los perfiles espectrales y los valores de SC. Se obtuvieron modelos con el espectro completo de onda y modelos optimizados, con las longitudes de onda relevantes. Los resultados obtenidos con el modelo PLSR optimizado mostraron valores de R2 superiores a 0,89 y RMSEC menores de 4,86x10-15. Por tanto; el método NIR - PLSR mostró potencialidades para la determinación de citral en microcápsulas.
\end{abstract}

Palabras clave: Absorbancia; Eficiencia de encapsulación; Microencapsulación; Perfil espectral; Reflectancia. 


\section{Introducción}

El citral (3, 7-Dimetil-2, 6-octadienal), es un compuesto de amplio uso en las industrias de bebidas, refrescos, panificación, golosinas, farmacia, perfumería, cigarrillos y otros [1,2]; principalmente debido al amplio espectro antimicrobiano in vitro y la capacidad antioxidante que ha demostrado [3-6].

Sin embargo, estas estructuras son susceptibles a degradación frente a altas concentraciones de oxígeno, cambios de $\mathrm{pH}$, procesos de deshidratación, presencia de luz y humedad durante el almacenamiento, dando como resultado la pérdida de su actividad [6]. En este sentido, investigadores como Lu et al. [4] y Yang et al. [6] indican que una alternativa para proteger las actividades del citral es la microencapsulación.

Las técnicas para microencapsular citral y otros derivados del aceite de limón en la industria alimentaria, en su mayoría se basan en sistemas coloidales blandos, siendo principalmente las emulsiones de aceite en agua, nanoemulsiones, complejos moleculares, liofilización, secado por atomización y secado por lecho fluidizado. Sin embargo, el secado por atomización es el método más utilizado en alimentos [2,7] y su eficiencia se evalúa principalmente mediante la cantidad del compuesto activo que es atrapado dentro de la matriz (Eficiencia de encapsulación - EM) [1]. La cantidad de compuesto activo que queda fuera del agente encapsulante, viene a ser el activo superficial; con la cuantificación de este activo superficial se puede inferir el cálculo de la EM [8].

Se ha demostrado que el parámetro EM se encuentra relacionado con los parámetros de formulación y de proceso como: tipo de material de pared utilizado, concentración de sólidos en la solución de alimentación y temperatura del aire de entrada al secador [7]. En la literatura se reporta que para aceites esenciales, el activo superficial o encapsulado ha sido medido usando las siguientes técnicas de análisis:

Gravimétrico $(G v)$. Es la de mayor uso en microencapsulación de aceites, la cuál ha sido aplicada por Chang et al. [9] en encapsulación de aceite de canola y nuez; Sharif et al. [10] en emulsiones de $\beta$-caroteno, eugenol y aceite de semilla de lino; Timilsena et al. [11] en aceite de semilla de chia (Salvia hispanica L.) y Velmurugan et al. [12] en aceite esencial de naranja (Citrus sinensis) y lavanda (Lavandula angustifolia).

Espectrofotometría ultravioleta (EUV). Se ha empleado en aceite esencial de jengibre [13]; en eugenol recubietas con $\beta$-ciclodextrina [14]; aceite esencial de albahaca [15] y en aceite de semilla de rosa [16].

Cromatografía líquida de alta eficiencia (HPLC). Ha sido aplicada en tebuconazol y resveratrol [17].

Cromatografía de gases (GC). Se ha reportado su aplicación por Campelo et al. [1], para aceite esencial de lima.

Cromatografía de gases con espectrómetrometría de masas (GC-MS). Se ha empleado en aceite de hojas de canela (Canela verum), aceite de orégano (Origanum vulgare) [18] y aceite esencial de naranja [12].

En el caso de citral se han usado diferentes técnicas, para medir el activo superficial: GC en diversos trabajos [19-22]; GC con microextracción en fase sólida (solid phase microextraction gas chromatography: SPMEGC) [23]; GC-MS [24,25]; HPLC [26]; EUV [4,27]. El uso de estas técnicas en su mayoría demandan de tiempos prolongados, preparación previa de muestras, alto costo y uso de reactivos químicos perjudiciales para el ambiente.

Por otro lado, una técnica de amplio uso y gran potencial, es la espectroscopía de infrarrojo cercano (NIR), la cual ha mostrado una aplicación potencial para el análisis en industrias de polímeros, textiles, en el control de procesos industriales, en las ciencias biomédicas [28] y principalmente más utilizado en las industrias agrícolas y alimentarias [29]. Sin embargo, existen relativamente pocos trabajos sobre la aplicación de la espectroscopía NIR para la cuantificación de citral, en aceites esenciales de limón y naranja [30].

La espectroscopía NIR tiene entre sus ventajas: permite realizar mediciones de varios componentes en forma rápida y simultánea, no es destructiva, es de bajo costo, no requiere reactivos, no requiere preparación intensiva de la muestra [29]. Esta técnica tiene como principio medir la respuesta, principalmente vibraciones de enlaces $-\mathrm{CH},-\mathrm{OH},-\mathrm{SH}$ y $-\mathrm{NH}$, de una muestra cuando es sometida a energía electromagnética entre 800 a 2500 nm conformando así huellas o perfiles espectrales [28]. Sin embargo, por la cantidad de información contenida, el análisis de estos perfiles espectrales requiere el uso de herramientas quimiométricas como análisis de componentes principales (ACP), coeficiente de correlación (CC), redes neuronales artificiales (RNA); regresión multilineal múltiple (RLM), regresión parcial por mínimos cuadrados (PLSR), entre otras [28,31].

En función de lo anteriormente expuesto, esta 
investigación tiene como objetivo predecir el contenido superficial de citral en micropartículas mediante espectroscopía NIR y regresión por mínimos cuadrados parciales.

\section{Parte Experimental}

\section{Reactivos}

Los reactivos usados en la producción y análisis de las microcápsulas fueron lecitina de soya (Epikuron ${ }^{\circledR}$ 145 V, Cargill, Alemania), dextrina (Amisol ${ }^{\circledR}$ 4810, Ingredion, Perú), citral mixture cis y trans $\geq 96 \%$ (Sigma - Aldrich, Alemania) y citral analytical estandard (Sigma Aldrich, Alemania).

\section{Encapsulación}

La encapsulación de citral mediante secado por atomización, se realizó variando la relación citral:dextrina (Ct:Dx) (1:5 y 1:20) en la solución de alimentación y la temperatura del aire de entrada al secador (TAI) al secador de atomización $\left(120-200{ }^{\circ} \mathrm{C}\right)$; generando un total de doce tratamientos experimentales.

Para cada tratamiento se consideró $100 \mathrm{~g}$ de solución de alimentación. La emulsión (Citral y lecitina de soya - LS) se preparó disolviendo $1 \mathrm{~g}$ de LS en $30 \mathrm{~mL}$ de agua destilada a $50^{\circ} \mathrm{C}$, se mantuvo en agitación constante (350 rpm) por 15 minutos, se enfrió a $30^{\circ} \mathrm{C}$, luego se agregó $1 \mathrm{~g}$ de citral mixture y se homogenizó por 3 minutos a $15000 \mathrm{rpm}$ con un homogeneizador Polytron PT 2100 (Kinematica AG, Suiza). Paralelamente, la dextrina - Dx (5 - 20 g) se disolvió en agua destilada (48 - 63 g) a 50 ${ }^{\circ} \mathrm{C}$, manteniendo en agitación constante por 3 horas, se enfrió a $30^{\circ} \mathrm{C}$ y luego se mezcló con la emulsión. La mezcla resultante se homogenizó a 15000 rpm por 3 minutos y se alimentó inmediatamente al secador mini Spray.

\section{Secado por atomización}

Las dispersiones obtenidas fueron alimentadas a un secador mini Spray-Dryer Büchi modelo B-290 (Suiza), con alimentación y flujo de aire de secado en configuración co-corriente. Las condiciones de proceso en el secador fueron: temperatura de alimentación de la solución de $42 \pm 2,5^{\circ} \mathrm{C}$, flujo de alimentación de $5 \mathrm{~mL} / \mathrm{min}$, presión de atomización de 50 mbar, porcentaje de aspiración de $100 \%$, flujo de aire de $600 \mathrm{~L} / \mathrm{h}$ y temperatura de entrada del aire al secador que varió entre 120 y $200^{\circ} \mathrm{C}$. Las micropartículas se almacenaron a $-20^{\circ} \mathrm{C}$ en tubos falcón de $45 \mathrm{~mL}$ hasta su análisis.

\section{Determinación del contenido superficial de citral (SC)}

Para la determinación del contenido SC se pesaron 0,2 g de micropartículas (mcp), se adicionaron $2 \mathrm{~mL}$ de hexano, luego se agitaron manualmente con tres movimientos de inversión, se centrifugaron a 2000 rpm por 1 minuto, finalmente, los sobrenadantes se transfirieron a viales ámbar de $2 \mathrm{~mL}$ para la lectura de sus absorbancias en el cromatógrafo de gases [32,33]. La concentración SC se calculó de acuerdo a la Ec. (1) [8].

$$
S C(\text { mgcitral/gmcp })=\frac{C C s}{W_{m c p}}
$$

donde CCs es la concentración del citral superficial en el solvente (mg citral en $2 \mathrm{~mL}$ de hexano), y es la masa de la micropartícula de citral en (g).

\section{Cromatografía de gases}

La determinación del contenido de citral superficial de las micropartículas se realizó en un cromatógrafo de gases (7890A, Agilent Technologies, USA) equipado con una columna capilar de sílice fundido DB-5MS 30m x 0,25 mm x 0,25 $\mu \mathrm{m}$ (J\&W Scientific, USA) y un detector de ionización de llama (FID- flame ionization detector). La temperatura del inyector y el detector fue de $250{ }^{\circ} \mathrm{C}$. Como gas transportador se utilizó nitrógeno a un flujo de $1,11 \mathrm{~mL} / \mathrm{min}$; la inyección del volumen de muestra fue $1 \mu \mathrm{L}$; la temperatura de la columna se programó inicialmente a $60{ }^{\circ} \mathrm{C}$ por $5 \mathrm{~min}$, luego se calentó hasta $120{ }^{\circ} \mathrm{C}$ a una razón de $5^{\circ} \mathrm{C} / \mathrm{min}$, posteriormente, hasta $250{ }^{\circ} \mathrm{C}$ a un ratio de $10{ }^{\circ} \mathrm{C} / \mathrm{min}$; siguiendo la metodología propuesta por Ruktanonchai et al. [24]. La cuantificación de citral se realizó utilizando una curva de calibración (1 - $2000 \mu \mathrm{g} / \mathrm{mL}, \mathrm{R}^{2}=0$,999). Los análisis se realizaron por duplicado para cada tratamiento y los resultados se expresaron en mg citral/g de mcp [8].

\section{Espectroscopía NIR}

Las microcápsulas obtenidas en los doce tratamientos se dividieron en seis partes, cuatro para modelado y dos para validación, obteniéndose un total de 72 muestras a las cuales se les determinó su perfil espectral.

La medición de los espectros de absorbancia se realizó de acuerdo a los trabajos de Escudero et al. [34] y Steuer et al. [35]; usando un espectrómetro infrarrojo cercano Unity Scientific NIRS (SpectraStar 2500XL, EE.UU.) equipado con lámpara halógena de tungsteno como fuente de luz y detector InGaAs (Indio - Galio - Arsénico) en el rango de 1100 y $2500 \mathrm{~nm}$, con una resolución de $1 \mathrm{~nm}$. Las mediciones se realizaron en el modo reflectancia aplicado directamente a las micropartículas sin tratamiento ni 
manipulación previa, utilizando una cubeta de cuarzo de 3,5 cm de diámetro interno y 1,0 cm de espesor, donde se adicionaron $2,3 \pm 0,5 \mathrm{~g}$ de micropartículas.

\section{Pretratamiento de los perfiles espectrales}

Los espectros se pretrataron utilizando un filtro de Savitzky-Golay de segundo orden de acuerdo con los estudios de Vásquez et al. [36] y Castro et al. [37]. Posteriormente, se dividieron aleatoriamente en conjuntos de datos de calibración y validación, cuarenta y ocho y veinticuatro perfiles espectrales, respectivamente. Todos los datos se guardaron en hojas de cálculo Excel para la etapa de modelado.

\section{Modelado}

Para modelar las relaciones entre los perfiles espectrales y los parámetros del contenido de SC se utilizó el método de regresión de mínimos cuadrados parciales (PLSR) [28,31,36].

PLSR es un método estadístico que transforma variables de entrada $X$ en la salida $Y$. En este trabajo, $X$ es una matriz de absorbancia, donde $n$ es la longitud de onda y $m$ es el número de observaciones, $Y$ son los valores de la variable respuesta (contenido SC) de las micropartículas. PLSR descompone $X$ e $Y$ al proyectarlos en nuevas direcciones con la restricción de que la descomposición describe cómo las variables cambian juntas tanto como sea posible. Después de la descomposición variable, se realiza un paso de regresión en el que las $X$ y las $Y$ descompuestas se usan para calcular un modelo de regresión llamado modelo completo (Ec. 2) [36]. sobre la base de los pesos $w$ de carga según Mehmood et al. [38]. Las longitudes de onda se vincularon a los pesos de carga absolutos para el modelo PLSR y se seleccionaron de acuerdo con su valor y capacidad para explicar $Y$, lo que redujo el error cuadrático medio (RMSE). Posteriormente, se construyeron modelos simplificados u optimizados utilizando solo las longitudes de onda más relevantes [36]. Las funciones usadas para el modelado se implementaron en script de Matlab 2015‥

\section{Evaluación del modelo}

Para la medición de la robustez y predictibilidad de técnicas basadas en análisis multivariados es común el uso del coeficiente de determinación $\left(R^{2}\right)$ y el RMSE. Estas medidas pueden ser calculadas usando conjunto de muestras para calibración (modelado), obteniendo $R_{c}^{2}$, $R M S E_{c}$; y para validación, obteniendo $R_{v}^{2}, R M S E_{v}$; usando las Ecs. (4) y (5) (39).

$$
\begin{gathered}
R_{C, v}^{2}=\frac{\sum_{i=1}^{n_{c, v}}\left(\hat{y}_{i}-y_{i}\right)^{2}}{\sum_{i=1}^{n_{C, v}}\left(\hat{y}_{i}-y_{c, v}\right)^{2}} \\
\operatorname{RMSE}_{C, v}=\sqrt{\frac{1}{n_{c, v}} \sum_{i=1}^{n_{c, v}}\left(\hat{y}_{i}-y_{c, v}\right)^{2}}
\end{gathered}
$$

donde e son los valores de los atributos de calidad de las muestras para predicción y referencia, respectivamente. e son los valores promedio de los conjuntos de datos de calibración y validación, respectivamente. e son los números de muestras de los conjuntos de datos de calibración y validación, respectivamente.

$$
\underbrace{\left|\begin{array}{c}
Y_{1} \\
\vdots \\
Y_{n-1} \\
Y_{n}
\end{array}\right|}_{\text {Contenido SC }}=\underbrace{\left|\begin{array}{c}
\beta_{1} \\
\vdots \\
\beta_{n-1} \\
\beta_{n}
\end{array}\right|}_{\text {Coeficientes Beta }}
$$

Esta ecuación se puede reescribir como en la Ec. (3).

$$
Y=\beta . X+e
$$

donde $Y$ es la matriz de valores de la variable respuesta, $\beta$ es la matriz de coeficientes beta, $X$ es la matriz de datos de absorbancia, y $e$ es el error del modelo.

La metodología fue adaptada de Vásquez et al. [36], los modelos PLSR completos se construyeron usando todas las longitudes de onda en un perfil espectral, y la relevancia de cada longitud de onda en el modelo se evaluó
Perfil de absorbamcia de "m" muestras

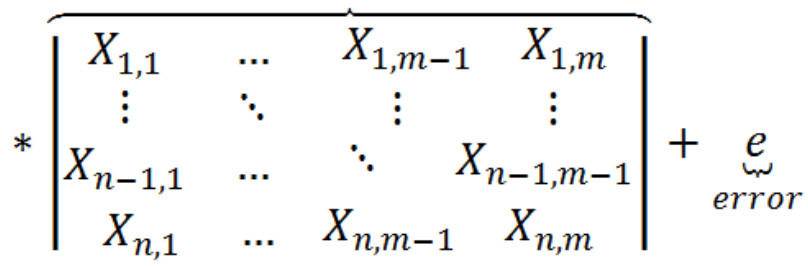

La validación del modelo se realizó con el conjunto de datos para validación mencionadas en los apartado anteriores; comparando los valores reales y predichos de las variables respuesta. Se determinaron los estadísticos $\mathrm{R}^{2}$ y RMSE. Finalmente, dichos estadísticos permitieron comparar los modelos completo y optimizado.

\section{Resultados y Discusión}

\section{Análisis de referencia}

Según los análisis de referencia de las 
micropartículas, realizado mediante cromatografía de gases, la concentración del citral superficial varió entre 0,17 y $1,28 \mathrm{mg}$ citral/g mcp.

\section{Perfiles espectrales del contenido superficial de citral}

La Figura 1 muestra los perfiles espectrales medios para las micropartículas con diferentes concentracones de citral; en esta figura se observa picos relacionados con las bandas de absorbancia en la región de sobretonos de 1206 a 1761 nm y la región de combinación de 1934 a $2490 \mathrm{~nm}$.

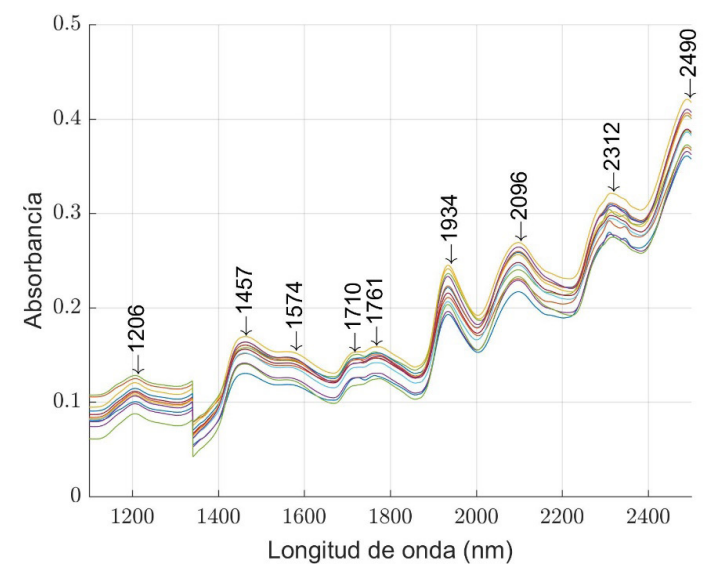

Figura 1. Perfiles espectrales medios de las micropartículas.

Los picos de absorción entre 2096 y 2312 nm están dentro de los rangos correspondientes a las interacciones $\mathrm{C}-\mathrm{H}$ flexión- extensión y $\mathrm{C}=\mathrm{O}$ extensión, posiblemente debido a la presencia del grupo aldehído [35,40]. Los picos 1206, 1710 y $1761 \mathrm{~nm}$ corresponden a la interacción $\mathrm{C}$-H extensión, probablemente por la presencia de dextrina, polímero usado en este estudio para la encapsulación del citral; estos resultados obtenidos coinciden con los reportados por Magwaza et al. [41] y Shenk et al. [42].

Igualmente; los picos 1457 y 1934 corresponden a las bandas de vibración del estiramiento de $\mathrm{O}-\mathrm{H}$ extensión y $\mathrm{O}-\mathrm{H}$ combinación, respectivamente, principalmente debido a la presencia de agua; además se observó un pequeño pico a $1574 \mathrm{~nm}$, que está dentro de la banda vibratoria de $C$ - $H$ extensión, asociado a los lípidos, probablemente debido a la presencia de lecitina de soya utilizada como emulsificante en la preparación de las micropartículas [29].

Estos perfiles indicaron una correspondencia entre los cambios de los espectros y el contenido de SC en las micropartículas, lo que permite el análisis de patrones y la implementación de modelos de predicción.

\section{Modelado PLSR}

En las siguientes subsecciones se muestran los principales resultados de la etapa de modelado.

\section{a) PLSR completo}

Los resultados de los modelos PLSR completos se muestran en la Figura 2 y la subsección 3.4; en ambas figuras, se observó que el $\mathrm{R}^{2}$ estuvo cerca de 1.0.

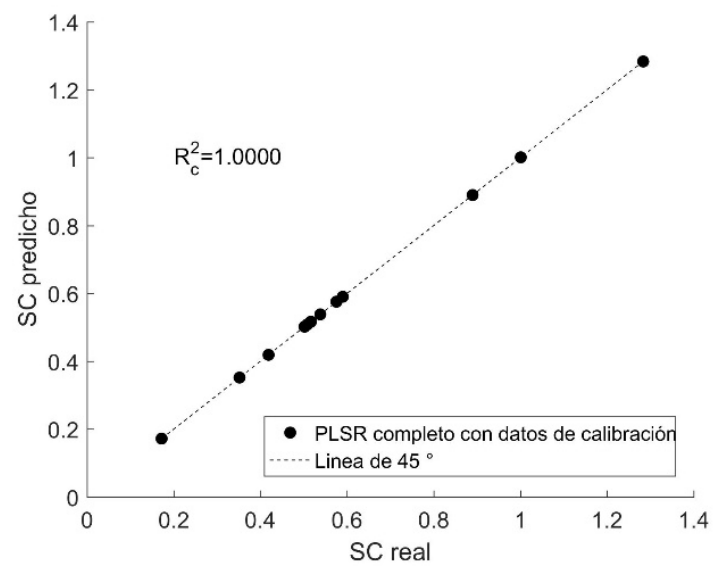

(a)

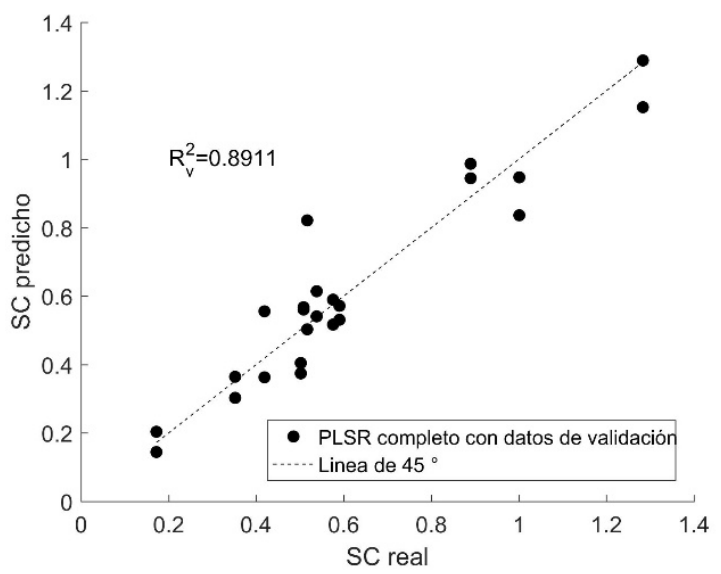

(b)

Figura 2. Resultados de los modelos PLSR completos utilizando (a) calibración; (b) validación.

Sin embargo, en los resultados la Figura $2 b$ presentó una mayor dispersión, con respecto a la Figura 2a. En este caso, de acuerdo con Esposito et al. [43], Maaten et al. [44] y Vega et al. [45], estos resultados pueden deberse a la alta colinealidad y la superposición de los perfiles 
espectrales que introducen ruido en el modelo, siendo necesario determinar previamente la relevancia de cada longitud de onda antes de ser utilizada en el modelo.

\section{b) PLSR optimizado}

La Figura 3 resume los resultados de esta etapa. La Figura 3a muestra el número óptimo de variables latentes (LV) (longitudes de onda), los cuales fueron 17, obtenidos a través de la minimización de RMSE y la maximización de $\mathrm{R}^{2}$, utilizando PLSR con validación cruzada para el contenido de SC según lo propuesto por Liu et al. [46].

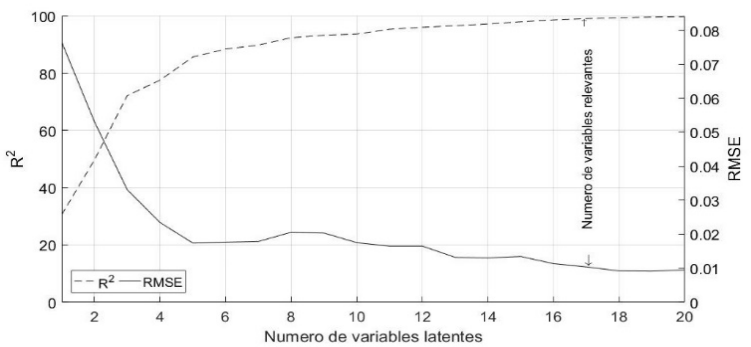

(a)

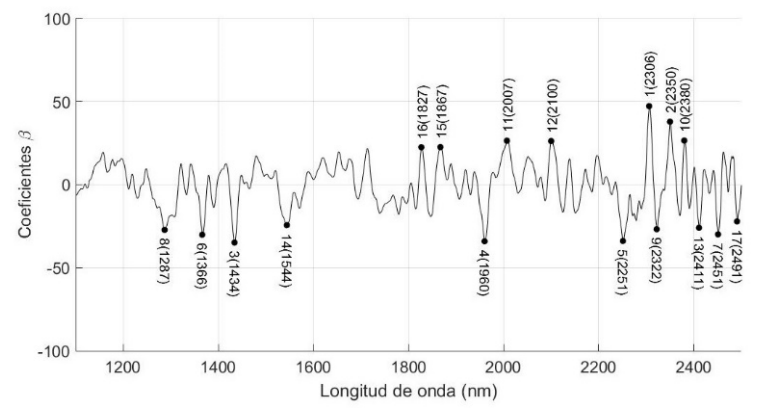

(b)

Figura 3. (a) Variables latentes (LV) para el modelado PLSR y (b) $\beta$-coeficientes y longitudes de onda relevantes (RW) para el modelo PLSR.

La Figura $3 \mathrm{~b}$ muestra los $\beta$-coeficientes del modelo PLSR, que representa la relevancia de cada longitud de onda y las longitudes de onda relevantes (marcadas con puntos negros), que se distribuyeron principalmente en el rango de [2251 -2491] nm, y otros dos rangos [1287 - 1544] nm y [1827 - 2100] nm.

Igualmente, se observó un mayor $\beta$-coeficiente a $2306 \mathrm{~nm}$ para citral causado por la combinación de las frecuencias de $\mathrm{C}=\mathrm{O}$ y $\mathrm{C}$ - $\mathrm{H}$ extensión del grupo aldehído que está contenido en citral. Los picos negativos (2251 y $2322 \mathrm{~nm}$ ) podría deberse a que los picos de absorbancia se invierten en el cálculo del $\beta$-coeficiente [40]. Estos resultados están dentro de los rangos reportados por
Steuer et al. [35], en la evaluación del espectro NIR del limoneno puro, en la que obtuvieron picos predominantes entre las áreas de 2250 y $2360 \mathrm{~nm}$. Por otro lado, los resultados del presente estudio fueron ligeramente superiores a los reportados por Wilson et al. [40] obtenidos en la evaluación del espectro NIR de citral en aceites esenciales de hierva de limón o limoncillo (Cymbopogon citratus) y limón (Citrus limon), observaron que el grupo funcional aldehídico $C$ - $H$ (contenido en el citral) exhibía bandas características de combinación entre 2210 y 2250 $\mathrm{nm}$; además, un incremento en la absorbancia alrededor de $2212 \mathrm{~nm}$ con el incremento del contenido de citral; esta diferencia en los resultados podría deberse a la presencia de dextrina y lecitina de soya como parte de la formulación de las micropartículas.

Finalmente, las longitudes de onda relevantes se usaron para construir los modelos optimizados PLSR, que resultaron del uso del conjunto de datos de calibración (Figura 4a y Figura 4b). En ambas figuras, se observaron que los $\mathrm{R}^{2}$ fueron 0,97 y 0,94 para calibración y validación,

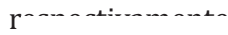

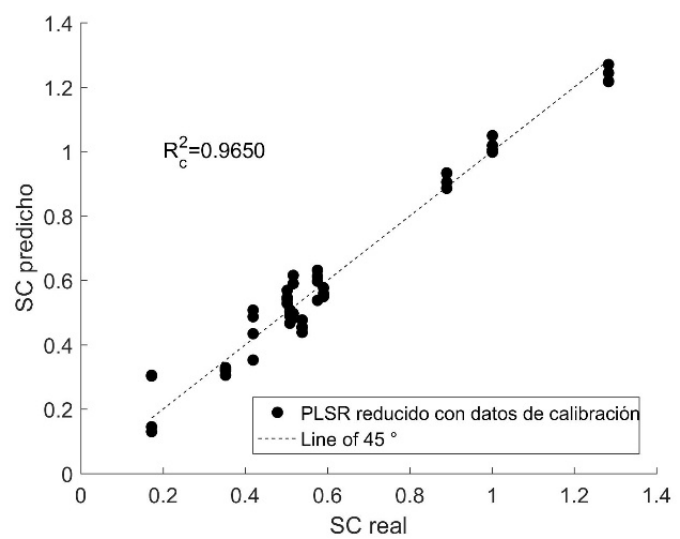

(a)

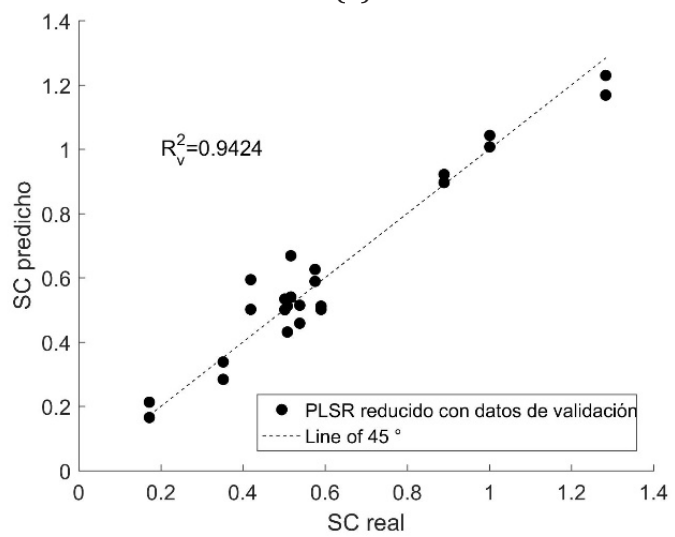

(b)

Figura 4. Resultados de los modelos PLSR optimizados utilizando (a) calibración; y (b) validación. 


\section{Comparación de modelos}

Las medidas estadísticas de los modelos PLSR (completos y optimizados) utilizando el conjunto de datos de calibración y validación se muestran en la Tabla 1.

\section{Referencias Bibliográficas}

[1] Campelo P.H., do Carmo E.L., Zacarias R.D., Yoshida M.I., Ferraz V.P., de Barros Fernandes R.V., Botrel D.A. and Borges S.V.. Effect of dextrose equivalent on physical and chemical properties of lime essential

Tabla 1. Medidas estadísticas para los modelos PLSR usados en la determinación del contenido superficial de citral

\begin{tabular}{lcccc}
\hline \multicolumn{1}{c}{ Modelos } & $R M S E_{c}$ & $R M S E_{v}$ & $R_{c}^{2}$ & $R_{v}^{2}$ \\
\hline Completo & $5,64 \times 10^{-15}$ & $6,38 \times 10^{-4}$ & 1,0000 & 0,8911 \\
Optimizado & $4,86 \times 10^{-15}$ & $2,20 \times 10^{-3}$ & 0,9650 & 0,9424 \\
\hline
\end{tabular}

Los modelos PLSR produjeron resultados aceptables con altos valores de $\mathrm{R}^{2}$ superiores a 0,89 , y con valores bajos de RMSE inferiores a 6,38 $\times 10^{-4}$, resultados similares a los obtenidos por Juliani et al. [47], en la evaluación de las estadísticas de correlación NIR para la composición de aldehídos de aceites esenciales en hoja de canela (Cinnamomum zeylanicum), hojas y brotes de clavo de olor (Syzygium aromaticum). Además, estos resultados fueron similares a los obtenidos por Steuer et al. [35] y Schulz et al. [48], para limoneno en aceite de cítricos; y citral en aceites esenciales de hierba de limón y limón. Finalmente, los resultados del presente estudio estuvieron dentro de los rangos obtenidos por Schulz et al. [49], quienes evaluaron la composición química de los aceites esenciales de tomillo, orégano y manzanilla, utilizando espectroscopía FT-IR y NIR; en todos estos estudios reportaron $\mathrm{R}^{2}$ mayor a 0,90 .

\section{Conclusiones}

El método NIR-PLSR fue capaz de predecir el contenido superficial de citral en las micropartículas, con $\mathrm{R}^{2}$ superiores a 0,89. Asimismo, al generar el modelo optimizado se observa que la robustez del modelo mejora reduciendo el valor RMSE e incrementaando el $\mathrm{R}^{2}$. En consecuencia, esta técnica permite inferir la eficiencia de encapsulación en micropartículas y muestra potencial para reemplazar los métodos tradicionales de evaluación de encapsulación de citral. En este contexto, la tecnología espectroscópica NIR junto a los métodos quimiométricos, por su rapidez y bajo costo, tendrá cada vez más importancia en el futuro cercano.

\section{Agradecimientos}

El primer autor agradece el apoyo al Consejo Nacional de Ciencia y Tecnología (CONCYTEC) por financiar la presente investigación y otorgar la beca para cursar el doctorado en Ciencia de Alimentos dentro del programa doctoral de la Universidad Nacional Agraria La Molina - Lima. oil microparticles. Ind Crops Prod., Vol. 102, (2017) 105-114.

[2] Maswal M. and Dar A.A. Formulation challenges in encapsulation and delivery of citral for improved food quality. Food Hydrocoll., Vol. 37, (2014) 182195.

[3] Fancello F., Petretto G.L., Zara S., Sanna M.L., Addis R., Maldini M., Foddai, M., Rourke J.P., Chessa M. and Pintore, G. Chemical characterization, antioxidant capacity and antimicrobial activity against food related microorganisms of Citrus limon var. pompia leaf essential oil. LWT - Food Sci Technol., Vol. 69, (2016) 579-585.

[4] Lu W.C., Huang D.W., Wang C.C.R., Yeh C.H., Tsai J.C., Huang Y.T. and Li P.H. Preparation, characterization, and antimicrobial activity of nanoemulsions incorporating citral essential oil. J Food Drug Anal., Vol. 26, $\mathrm{N}^{\circ}$. 1, (2018) 82-89.

[5] Saddiq A.A. and Khayyat S.A. Chemical and antimicrobial studies of monoterpene: Citral. Pestic Biochem Physiol., Vol. 98, N. 1, (2010) 89-93.

[6] Yang Y., Cui S., Gong J., Miller S.S., Wang Q. and Hua Y. Stability of citral in oil-in-water emulsions protected by a soy protein-polysaccharide Maillard reaction product. Food Res Int., Vol. 69, (2015) 357-363.

[7] Botrel D.A., de Barros Fernandes R.V. and Borges S.V. Chapter 12: Microencapsulation of Essential Oils Using Spray Drying Technology. In: L. Sagis (ed), Microencapsulation and Microspheres for Food Applications. Academic Press, San Diego, USA, (2015), 235-251.

[8] García P., Vega J., Jimenez P., Santos J. and Robert P. Alpha-tocopherol microspheres with cross-linked and acetylated inulin and their release profile in a 
hydrophilic model. Eur J Lipid Sci Technol., Vol. 115, $\mathrm{N}^{\circ} 7,(2013)$ 811-819.

[9] Chang C., Varankovich N. and Nickerson M.T. Microencapsulation of canola oil by lentil protein isolate-based wall materials. Food Chem., Vol. 212, (2016) 264-273.

[10] Sharif H.R., Goff H.D., Majeed H., Shamoon M., Liu F., Nsor-Atindana J, Haider, J., Liang R. and Zhong F. Physicochemical properties of $\beta$-carotene and eugenol co-encapsulated flax seed oil powders using OSA starches as wall material. Food Hydrocoll., Vol. 73, (2017) 274-283.

[11] Timilsena Y.P., Adhikari R., Barrow C.J. and Adhikari B. Microencapsulation of chia seed oil using chia seed protein isolate-chia seed gum complex coacervates. Int J Biol Macromol., Vol. 91, (2016) 347-357.

[12] Velmurugan P., Ganeshan V., Nishter N.F. and Jonnalagadda R.R. Encapsulation of orange and lavender essential oils in chitosan nanospherical particles and its application in leather for aroma enrichment. Surfaces and Interfaces, Vol. 9, (2017) 124-132.

[13] De Barros Fernandes R.V., Borges S.V., Silva E.K., da Silva Y.F., de Souza H.J.B., do Carmo E.L., de Oliveira C.R., Yoshida M.I. and Botrel D.A. Study of ultrasoundassisted emulsions on microencapsulation of ginger essential oil by spray drying. Ind Crops Prod., Vol. 94, (2016) 413-423.

[14] Piletti R., Bugiereck A.M., Pereira A.T., Gussati E., Dal Magro J., Mello J.M.M., Dalcanton F., Ternus R.Z., Soares C., Riella H.G., et al. Microencapsulation of eugenol molecules by $\beta$-cyclodextrine as a thermal protection method of antibacterial action. Mater Sci Eng C. Vol. 75, (2017) 259-271.

[15] Sutaphanit P. and Chitprasert P. Optimisation of microencapsulation of holy basil essential oil in gelatin by response surface methodology. Food Chem., Vol. 150, (2014) 313-320.

[16] Yao Z.C., Chang M.W., Ahmad Z. and Li J.S. Encapsulation of rose hip seed oil into fibrous zein films for ambient and on demand food preservation via coaxial electrospinning. J Food Eng., Vol. 191, (2016) 115-123.

[17] Salgado M., Rodríguez-Rojo S., Alves-Santos F.M. and Cocero M.J. Encapsulation of resveratrol on lecithin and $\beta$-glucans to enhance its action against Botrytis cinerea. J Food Eng., Vol. 165, (2015) 13-21.

[18] Munhuweyi K., Caleb O.J., Van Reenen A.J. and Opara U.L. Physical and antifungal properties of $\beta$-cyclodextrin microcapsules and nanofibre films containing cinnamon and oregano essential oils. LWT - Food Sci Technol., Vol. 87, (2018) 413-422.

[19] Sosa N., Zamora M.C., Chirife J. and Schebor C. Spray-drying encapsulation of citral in sucrose or trehalose matrices: physicochemical and sensory characteristics. Int J Food Sci Technol., Vol. 46, (2011) 2096-2102.

[20] Sosa N., Schebor C. and Pérez O.E. Encapsulation of citral in formulations containing sucrose or trehalose: Emulsions properties and stability. Food Bioprod Process., Vol. 92, N³, (2014) 266-274.

[21] Strassburger K., Startup W., Levey V., Mattingly T., Briggs J., Harrison J. and Wilson T. Chapter 11: Enhanced Stability of Citral in Juice Beverages by Applying Cyclodextrin Micro Emulsion Technology. In: N.C. Da Costa y R.J. Cannon (ed). Flavors in Noncarbonated Beverages. American Chemical Society, Washington DC, USA, (2010), 143-158.

[22] Bhandari B.R., Dumoulin E.D., Richard H.M.J., Noleau I. and Lebert A.M. Flavor Encapsulation by spray drying: Aplication to citral and linalyl acetate. J Food Sci., Vol. 57, N 1, (1992) 217-221.

[23] Yang X., Tian H., Ho C. and Huang Q. Inhibition of Citral Degradation by Oil-in-Water Nanoemulsions Combined with Antioxidants. J Agric Food Chem., Vol. 59, (2011) 6113-6119.

[24] Ruktanonchai U., Srinuanchai W., Saesoo S., Sramala I., Puttipipatkhachorn S. and Soottitantawat A. Encapsulation of Citral Isomers in Extracted Lemongrass Oil with Cyclodextrins: Molecular Modeling and Physicochemical Characterizations. Biosci Biotechnol Biochem., Vol. 75, N 12, (2011) 2340-2345.

[25] Yang X., Tian H., Ho C. and Huang Q. Stability of Citral in Emulsions Coated with Cationic Biopolymer Layers. J Agric Food Chem., Vol. 60, (2012) 402-409.

[26] Weisheimer V., Miron D., Silva C.B., Guterres S.S. and Schapoval E.E.S. Microparticles containing lemongrass volatile oil: Preparation, characterization and thermal stability. Pharmazie, 
Vol. 65, (2010) 885-890.

[27] Miron D., Battisti F., Caten C.S.T., Mayorga P. and Schapoval E.E.S. Spectrophotometric Simultaneous Determination of Citral Isomers in Cyclodextrin Complexes with Partial Least Squares Supported Approach. Curr Pharm Anal., Vol. 8, (2012) 401-408.

[28] Roggo Y., Chalus P., Maurer L., Lema-Martinez C., Edmond A. and Jent N. A review of near infrared spectroscopy and chemometrics in pharmaceutical technologies. J Pharm Biomed Anal., Vol. 44, (2007) 683-700.

[29] Kawano S. Chapter 12: Application to Agricultural Products and Foodstuff. In: H.W. Siesler, Y. Ozaki, S. Kawata y H.M. Heise (eds). Near-Infrared Spectroscopy: Principles, Instruments, Application. 3rd. ed. Wiley-VCH Verlag $\mathrm{GmbH}$, Weinheim, Germany, (2007), 269-287.

[30] López P., Gándara J. and Losada P. Infrared spectrophotometric determination of citral corrected for limonene interference in lemon and orange essential oils. Food Chem., Vol. 46, $\mathrm{N}^{\circ} 2$, (1993) 193-197.

[31] Heise H.M. and Winzen R. Chapter 7: Chemometrics in near-infrared spectroscopy. In: H.W. Siesler, Y. Ozaki, S. Kawata y H.M. Heise (eds). Near-Infrared Spectroscopy: Principles, Instruments, Application. 3rd. ed. Wiley-VCH Verlag $\mathrm{GmbH}$, Weinheim, Germany, (2007), 125-162.

[32] Vergara C., Saavedra J., Sáenz C., García P. and Robert P. Microencapsulation of pulp and ultrafiltered cactus pear (Opuntia ficus-indica) extracts and betanin stability during storage. Food Chem., Vol. 157, (2014) 246-251.

[33] Bustamante A., Masson L., Velasco J., Del Valle J.M. and Robert P. Microencapsulation of $\mathrm{H}$. pluvialis oleoresins with different fatty acid composition: Kinetic stability of astaxanthin and alpha-tocopherol. Food Chem., Vol. 190, (2016) 1013-1021.

[34] Escuredo O., González-Martín M.I., Wells-Moncada G., Fischer S. and Hernández-Hierro J.M. Amino acid profile of the quinoa (Chenopodium quinoa Willd.) using near infrared spectroscopy and chemometric techniques. J Cereal Sci. Vol. 60, Nº 1, (2014) 67-74.

[35] Steuer B., Schulz H. and Läger E. Classification and analysis of citrus oils by NIR spectroscopy. Food Chem., Vol. 72, N 1, (2001) 113-117.
[36] Vásquez N., Magán C., Oblitas J., Chuquizuta T., Avila-George H. and Castro W. Comparison between artificial neural network and partial least squares regression models for hardness modeling during the ripening process of Swiss-type cheese using spectral profiles. J Food Eng., Vol. 219, (2018) 8-15.

[37] Castro W., Prieto J.M., Guerra R., Chuquizuta T., Medina W.T., Acevedo-Juárez B. and Avila-George H. Feasibility of using spectral profiles for modeling water activity in five varieties of white quinoa grains. J Food Eng., Vol. 238, (2018) 95-102.

[38] Mehmood T., Liland K.H., Snipen L. and Sæbø S. A review of variable selection methods in Partial Least Squares Regression. Chemom Intell Lab Syst., Vol. 118, (2012) 62-69.

[39] Sun Y., Liu Y., Yu H., Xie A., Li X., Yin Y. and Duan X. Non-destructive prediction of moisture montent and freezable water content of purple-fleshed sweet potato slices during drying process using Hyperspectral Imaging Technique. Food Anal Methods. Vol. 10, N 5, (2017) 1535-1546.

[40] Wilson N.D., Ivanova M.S., Watt R.A. and Moffat A.C. The quantification of citral in lemongrass and lemon oils by near-infrared spectroscopy. J Pharm Pharmacol., Vol. 54, N 9, (2002) 1257-1263.

[41] Magwaza L.S., Opara U.L., Nieuwoudt H., Cronje P.J.R., Saeys W. and Nicolaï B. NIR Spectroscopy applications for internal and external quality analysis of citrus fruit-A Review. Food Bioprocess Technol., Vol. 5, N 2, (2012) 425-444.

[42] Shenk J.S., Workman J.J. and Weterhaus M.O. Chapter 16 - Aplication of NIR Spectroscopy to agricultural products. In: D.A. Burns y E.W. Ciurczak (eds). Handbook of Near-Infrared Analysis. 2th. editi. Taylor \& Francis Group, LLC, New York, USA, (2008), 347-386.

[43] Esposito V.V., Chin W.W., Henseler J. and Wang H. Handbook of partial least squares: Concepts, methods and aplications. Springer International Publishing, Berlin, Germany, 2010.

[44] Maaten L., Postma E. and Herik J. Dimensionality Reduction: A Comparative - Review. Tilbg Cent Creat Comput., (2009) 1-36.

[45] Vega-Vilca J.C. y Guzmán J. Regresión PLS y PCA como solución al problema de multicolinealidad en regresión múltiple. Rev Matemática Teoría y Apl., 
Vol. 18, $\mathrm{N}^{\circ} 1,(2011)$ 9-20.

[46] Liu D., Sun D.W. and Zeng X.A. Recent advances in wavelength selection techniques for Hyperspectral Image processing in the food industry. Food Bioprocess Technol., Vol. 7, (2014) 307-323.

[47] Juliani H.R., Kapteyn J., Jones D., Koroch A.R., Wang M., Charles D. and Simon J.E. Application of near-infrared spectroscopy in quality control and determination of adulteration of african essential oils. Phytochem Anal., Vol. 17, N² 2, (2006) 121-128.
[48] Schulz H., Schrader B., Quilitzsch R. and Steuer B. Quantitative analysis of various citrus oils by ATR/FT-IR and NIR-FT Raman spectroscopy. Appl Spectrosc., Vol. 56, N 1, (2002) 117-124.

[49] Schulz H., Quilitzsch R. and Krüger H. Rapid evaluation and quantitative analysis of thyme, origano and chamomile essential oils by ATR-IR and NIR spectroscopy. J Mol Struct., Vol. 661-662, (2003) 299-306. 


\section{REVISTA TECNICA}

DE LA FACULTAD DE INGENIERIA

UNIVERSIDAD DEL ZULIA

Vol. 42. N², Mayo -Agosto 2019, pp. 47 - 97

Esta revista fue editada en formato digital y publicada en Abril de 2019, por el Fondo Editorial Serbiluz, Universidad del Zulia. Maracaibo-Venezuela

www.luz.edu.ve

www.serbi.luz.edu.ve

produccioncientifica.luz.edu.ve 